\title{
Prevalence and Characteristics of Unruptured Cerebral Aneurysms in Ischemic Stroke Patients
}

\author{
Ji Hwa Kim, ${ }^{\text {a }}$ Sang Hyun Suh, ${ }^{\mathrm{b}}$ Joonho Chung, ${ }^{\mathrm{c}}$ Yeo-Jin Oh, ${ }^{\mathrm{a}}$ Sung Jun Ahn, ${ }^{\mathrm{b}}$ Kyung-Yul Lee ${ }^{\mathrm{a}}$ \\ ${ }^{a}$ Department of Neurology, Gangnam Severance Hospital, Yonsei University College of Medicine, Seoul, Korea \\ ${ }^{b}$ Department of Radiology, Gangnam Severance Hospital, Yonsei University College of Medicine, Seoul, Korea \\ 'Department of Neurosurgery, Gangnam Severance Hospital, Yonsei University College of Medicine, Seoul, Korea
}

Background and Purpose The prevalence of unruptured cerebral aneurysms (UCAs) in ischemic stroke patients is not clearly defined. This study aimed to measure the prevalence and characteristics of UCAs in patients with acute ischemic stroke (AIS) and to compare our findings with those of the general population. In addition, we investigated the factors associated with cerebral aneurysms in AIS patients.

Methods We retrospectively reviewed the brain magnetic resonance angiography images of 955 patients with AIS and 2,118 controls who had received a brain magnetic resonance angiography as part of a health check-up. We investigated the prevalence, size, location, and risk factors of the subjects in the context of UCAs.

Results UCAs were found in 74 patients with AIS (7.7\%) and in 79 who received a health check-up $(3.7 \%)$. The prevalence of UCAs was significantly higher in the AIS group than in the health check-up group (odds ratio 2.17, 95\% confidence interval 1.56-3.01). The mean aneurysm diameter was larger in the AIS group than in the health check-up group (3.75 mm vs. $3.02 \mathrm{~mm}, P=0.009$ ). UCAs were primarily located in the internal carotid artery in both groups, and aneurysms in the middle cerebral artery were particularly common in the AIS group. According to multivariate analysis, hypertension alone was associated with an increased prevalence of UCAs in stroke patients.

Conclusions This study identified a higher prevalence and larger size of UCAs in AIS patients than in the general population. Hypertension was an independent risk factor of UCA in AIS.

Keywords Cerebral aneurysm; Ischemic stroke; Prevalence; Magnetic resonance angiography

\author{
Correspondence: Kyung-Yul Lee \\ Department of Neurology, Gangnam \\ Severance Hospital, Yonsei University \\ College of Medicine, 211 Eonjuro, \\ Gangnam-gu, Seoul 06273, Korea \\ Tel: +82-2-2019-3325 \\ Fax: +82-2-3462-5904 \\ E-mail: kylee@yuhs.ac
}

Received: March 4, 2016 Revised: April 18, 2016 Accepted: May 3, 2016

The authors have no financial conflicts of interest.

\section{Introduction}

Cerebral aneurysms are abnormal focal pouch-like dilatations of the cerebral artery. The most dreaded complication of an unruptured cerebral aneurysm (UCA) is subarachnoid hemorrhage, which is associated with high morbidity and mortality. ${ }^{1,2}$ Thus, there is a great deal of interest in improving our ability to detect UCAs, as well as in investigating their natural history. ${ }^{3-5}$ Indeed, this interest has led to increased screening for UCAs and the exploration of novel treatment paradigms. ${ }^{6}$
The reported prevalence of UCAs ranges from 0.2 to $9 \%{ }^{7-17}$ and this wide range may be due to variable evaluation methods and demographics. Methodological differences include study design, study tools, imaging tools, patient selection, and aneurysm types. Likewise, differences in patient demographics may influence study results, with the prevalence of aneurysms differing between studies due to varying comorbidities.

While UCA and ischemic stroke share many risk factors, the prevalence of UCAs has not been investigated in patients with acute ischemic stroke (AIS). Thus, the purpose of this study was 
to evaluate the prevalence and characteristics of UCAs in AIS patients in comparison to a control population for the evaluation of comorbid factors related to the prevalence of UCAs in AIS patients.

\section{Methods}

\section{Patient selection}

This retrospective, cross-sectional study was conducted in a tertiary university hospital setting. Patients were classified into two groups for the study. The AIS group included patients with acute ischemic stroke who were admitted to the hospital and underwent brain magnetic resonance angiography (MRA) between January 2011 and December 2014. The health check-up (HC) group, which was used as the control group, consisted of individuals who underwent a brain MRA between January 2011 and December 2012 as part of a routine health examination.

Patient data including age, sex, past medical history (hypertension, diabetes, hyperlipidemia, previous stroke history, and smoking), family history of cerebrovascular disease (intracerebral hemorrhage, subarachnoid hemorrhage, or cerebral infarction), and follow-up results were obtained from medical records. In the AIS group, stroke subtypes were categorized by the Trial of Org 10172 in Acute Stroke Treatment (TOAST) criteria. ${ }^{18}$

The retrospective study design and protocol were approved by our institutional review board. The requirement for written informed consent from the patients was waived due to the retrospective nature of the design.

Image acquisition and interpretation

All 3-dimensional time-of-flight MRA examinations were performed using one of two 3.0T magnetic resonance imaging systems at our institution (Discovery MR750, GE Medical Systems, Milwaukee, WI, USA; Achieva, Philips Medical Systems, Best, The Netherlands). Three-dimensional time-of-flight MRA was performed with the following parameters for the Discovery MR750 instrument: repetition time/echo time, 23/2.5 ms; flip angle, 20 degrees; field of view, $210 \times 185 \mathrm{~mm} ; 4$ slabs (176 slices); slice thickness, $1.4 \mathrm{~mm}$; matrix, 416 $\times 224$; and acquisition time, 5 minutes and 9 seconds; and for the Achieva instrument: repetition time/echo time, 25/3.5 ms; flip angle, 20 degrees; field of view, $250 \times 198$ mm; 1 slab (170 slices); slice thickness, 1.4 mm; matrix, $832 \times 414$; and acquisition time, 6 minutes and 52 seconds.

UCAs were defined as abnormal focal outpouchings of the cerebral arteries. ${ }^{1}$ All radiologic reports from both groups were reviewed, and the MRA images of patients with UCA and with possible UCA according to those reports were independently reviewed by an experienced neurologist (J.H. Kim) and a neuroradiologist (S.H. Suh). In cases of discrepancy, a consensus inter- pretation was made. Patients with known UCAs prior to the MRA were also included regardless of treatment. Patients were excluded if they had fusiform, traumatic, or mycotic aneurysms, which have different etiologies, ${ }_{1}^{19}$ or aneurysms in an extradural location.

Aneurysm size was measured by the largest diagonal measurement. Aneurysm locations were classified as internal carotid artery (ICA), anterior cerebral artery, anterior communicating artery, middle cerebral artery (MCA), posterior communicating artery, and vertebrobasilar artery (including vertebral artery, basilar artery, posterior cerebral artery, and anterior and posterior cerebellar arteries). In addition, multiple aneurysms and the presence of daughter sacs were analyzed.

\section{Statistical analyses}

Differences between the two groups were evaluated using Student's $t$ test for continuous variables and the Chi-square test for categorical variables. Odds ratios (ORs) were derived from Chi-square test results with 95\% confidence intervals ( $\mathrm{Cls}$ ). The age-matched prevalence rate of UCA was analyzed by the Cochran-Mantel-Haenszel test. Linear regression analyses were performed to analyze the relationship between age and aneurysm size. The associations between UCA coexistence and various covariates in the AIS group were tested using logistic regression analysis. All $P$ values were 2 -sided and were considered statistically significant if they were 0.05 or less. Analyses were performed using SPSS version 20.0 (SPSS Inc., Chicago, IL, USA). The relationship between age and UCA prevalence was examined using the Cochran-Armitage trend test, which was conducted using SAS version 9.2 (SAS Institute, Cary, NC, USA).

\section{Results}

\section{Subject characteristics}

A total of 1,163 patients with suspected ischemic stroke were admitted to our hospital during the study period. Patients with transient ischemic attack $(n=203)$, and no MRA due to pacemaker implantation $(n=2)$, bionic ear $(n=1)$, and poor general condition $(n=2)$ were excluded from our analysis. Eventually, a total of 955 patients with AIS and 2,118 individuals who had undergone a brain MRA as part of a health check-up were enrolled in the study (Figure 1).

The baseline characteristics of the patients in each group are shown in Table 1. The mean age of the AIS group was significantly higher than that of the HC group $(64.9 \pm 14.1$ years vs. $53.9 \pm 9.6$ years, $P<0.001$ ). Other risk factors including hypertension, diabetes, hyperlipidemia, previous stroke history, smoking, and family history of cerebrovascular disease were also signifi- 
cantly more common in the AIS group.

\section{Prevalence of UCA}

UCAs were found in 74 patients in the AIS group (7.7\%; 38 men and 36 women; age 43 to 91 years, mean $67.2 \pm 11.2$ years) and 79 people in the control $(3.7 \% ; 35$ men and 44 women; age 37 to 76 years, mean $57.2 \pm 8.8$ years). Eight patients in the AIS group and 4 patients in the $\mathrm{HC}$ group had been diagnosed and treated for their UCA before this study. The prevalence of UCAs was significantly higher in the AIS group than in the $\mathrm{HC}$ group (OR 2.17, 95\% Cl 1.56-3.01, $P<0.001$ ).

In an age-matched comparison, the prevalence of UCAs was higher in the AIS group compared to the HC group based on the Cochran-Mantel-Haenszel test (OR 1.79, 95\% Cl 1.24-2.60, $P=0.002$ ). When categorized according to age by decade, a sig-

\section{Acute ischemic stroke group}

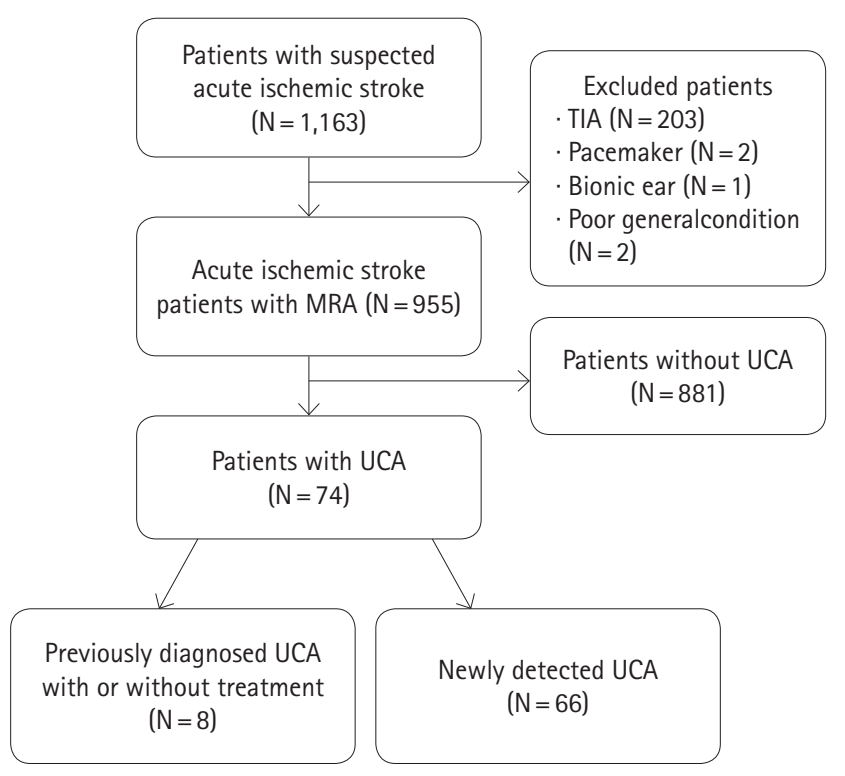

nificant difference in the prevalence between the two groups emerged only in the 7th decade (Table 2). The prevalence of aneurysm was higher in women than in men in both groups, and

Table 1. Baseline characteristics of subjects

\begin{tabular}{lccc}
\hline Characteristics & AIS $(n=955)$ & HC $(n=2,118)$ & $P$ value \\
\hline Age (mean \pm SD), (year) & $64.9 \pm 14.1$ & $53.9 \pm 9.6$ & $<0.001$ \\
Male & $586(61.4)$ & $1,188(56.1)$ & 0.06 \\
Hypertension & $600(62.8)$ & $622(29.4)$ & $<0.001$ \\
Diabetes & $289(30.3)$ & $241(11.4)$ & $<0.001$ \\
Hyperlipidemia & $134(14.0)$ & $189(8.9)$ & $<0.001$ \\
Previous stroke & $170(17.8)$ & $20(0.9)$ & $<0.001$ \\
Smokers & $417(43.7)$ & $710(33.5)$ & $<0.001$ \\
Family history of stroke & $94(9.8)$ & $44(2.1)$ & $<0.001$ \\
\hline
\end{tabular}

Values are number of patients (\%) unless otherwise specified. AIS, acute ischemic stroke; $\mathrm{HC}$, health check-up.

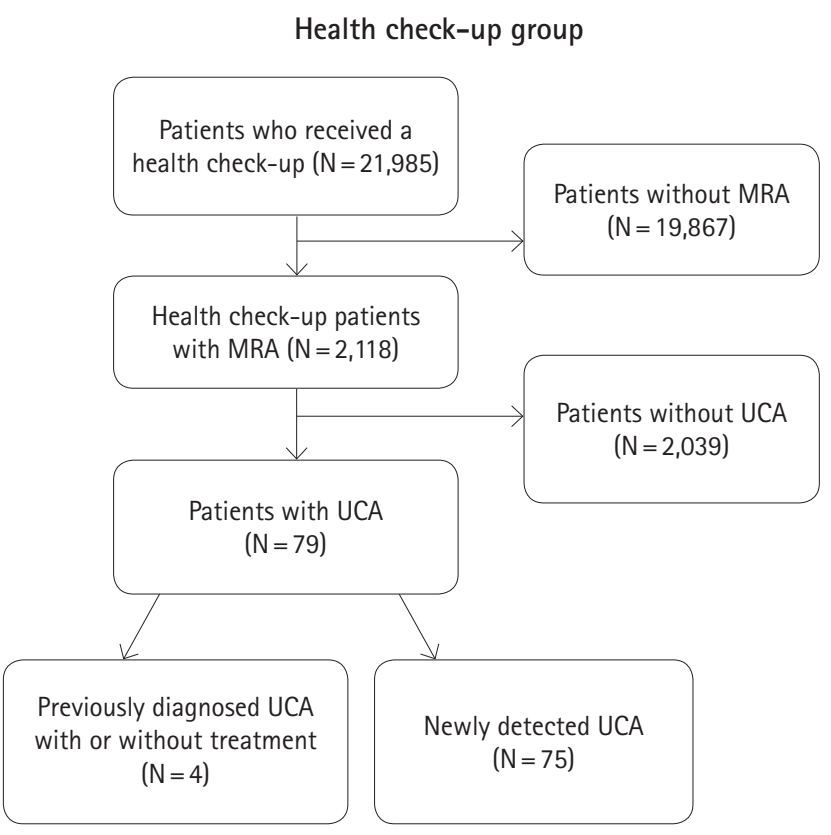

Figure 1. Flow diagram of participants. TIA, transient ischemic attack; MRA, magnetic resonance angiography; UCA, unruptured cerebral aneurysm.

Table 2. Age- and sex-specific prevalence of unruptured cerebral aneurysms

\begin{tabular}{|c|c|c|c|c|c|c|c|c|c|c|c|c|c|}
\hline \multirow{3}{*}{ Age (year) } & \multicolumn{6}{|c|}{ Acute ischemic stroke group } & \multicolumn{6}{|c|}{ Health check-up group } & \multirow{3}{*}{$P$ value } \\
\hline & \multicolumn{2}{|c|}{ Men } & \multicolumn{2}{|c|}{ Women } & \multicolumn{2}{|c|}{ Total } & \multicolumn{2}{|c|}{ Men } & \multicolumn{2}{|c|}{ Women } & \multicolumn{2}{|c|}{ Total } & \\
\hline & Pop & UCA & Pop & UCA & Pop & UCA & Pop & UCA & Pop & UCA & Pop & UCA & \\
\hline$<50$ & 99 & $3(3.0)$ & 38 & $3(7.9)$ & 137 & $6(4.4)$ & 371 & $8(2.2)$ & 282 & $8(2.8)$ & 653 & $16(2.5)$ & 0.212 \\
\hline $50-59$ & 146 & $8(5.5)$ & 42 & $3(7.1)$ & 188 & $11(5.9)$ & 522 & $16(3.1)$ & 390 & $20(5.1)$ & 912 & $36(3.9)$ & 0.240 \\
\hline $60-69$ & 162 & $14(8.6)$ & 75 & $11(14.7)$ & 237 & $25(10.5)$ & 224 & $8(3.6)$ & 209 & $11(5.3)$ & 433 & $19(4.4)$ & 0.002 \\
\hline$\geq 70$ & 179 & $13(7.3)$ & 214 & $19(8.9)$ & 393 & $32(8.1)$ & 71 & $3(4.2)$ & 49 & $5(10.2)$ & 120 & $8(6.7)$ & 0.598 \\
\hline Total & 586 & $38(6.5)$ & 369 & $36(9.8)$ & 955 & $74(7.7)$ & 1,188 & $35(3.0)$ & 930 & $44(4.7)$ & 2,118 & $79(3.7)$ & $<0.001$ \\
\hline$P$ value of trend ${ }^{+}$ & & 0.138 & & 0.932 & & 0.118 & & 0.325 & & 0.038 & & 0.024 & \\
\hline
\end{tabular}

Values are number of patients (\%) unless otherwise specified.

Pop, population; UCA, unruptured cerebral aneurysm.

${ }^{*} P$ value obtained by Chi-square test for comparisons between acute ischemic stroke group and health check-up group; ${ }^{+} P$ value of trend was calculated using the Cochran-Armitage trend test. 

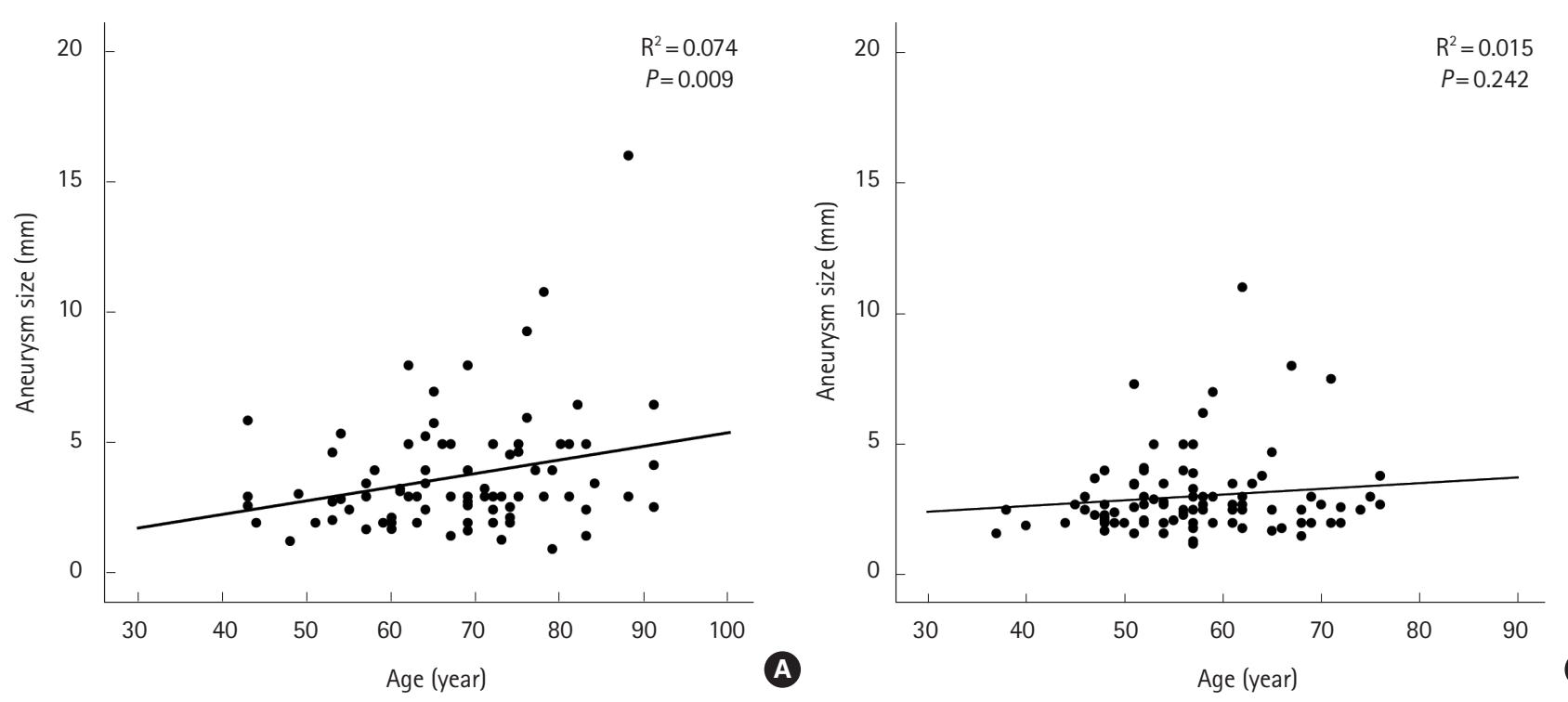

Figure 2. Relationship between the size of unruptured cerebral aneurysms and age in the acute ischemic stroke group (A) and the health check-up group (B). The regression lines are $y=0.052 x+0.233(A)$ and $y=0.022 x+1.768(B)$ where $y$ is aneurysm size $(\mathrm{mm})$ and $x$ is age (years).

increased significantly with increasing age among women in the HC group. On the other hand, there was a single peak incidence in the 7th decade in the AIS group (10.5\%) for both males $(8.6 \%)$ and females (14.7\%). Hypertension, previous stroke, and smoking were more common in AIS patients with UCAs than in HC patients with UCAs (Supplemental Table 1).

\section{Characteristics of UCA}

A total of 187 aneurysms were detected in 153 patients. Multiple aneurysms were noted in 13 patients $(17.6 \%)$ in the AIS group and 11 patients (13.9\%) in the HC group. The mean diameter of the UCAs was larger in the AIS group than in the HC group ( $3.75 \mathrm{~mm}$ vs. $3.02 \mathrm{~mm} ; P=0.009$ ). As shown in Figure 2 , the size of the UCAs increased with age in the AIS group $\left(R^{2}=\right.$ $0.074, P=0.009)$, but not in the HC group $\left(R^{2}=0.015, P=0.242\right)$. Aneurysms larger than $7 \mathrm{~mm}$ in diameter were more common in the AIS group than in the $\mathrm{HC}$ group ( $8.1 \%$ vs. $6.3 \%, P=0.67)$, and the mean diameter of UCAs was larger in women than in men in both groups (AIS group, $4.15 \pm 2.51 \mathrm{~mm}$ vs. $3.35 \pm 1.75 \mathrm{~mm} ; P=$ 0.082 and $\mathrm{HC}$ group, $3.12 \pm 1.70$ vs. $2.82 \pm 1.30 ; P=0.30$ ), but these differences were not significant.

Table 3 presents the incidence of UCAs in both groups according to their location. While the distal ICA was the most common site in both groups, MCA aneurysms were significantly more common in the AIS group compared to the HC group (OR 3.72, $95 \% \mathrm{Cl} 1.53-9.08)$. In analyzing the size of aneurysms by location, we found that the mean diameter was significantly larger in the AIS group than in the HC group for ICA only (Supplemental Table 2). Aneurysm daughter sacs were identified in two patients in each group.
Table 3. Distribution of unruptured cerebral aneurysm

\begin{tabular}{lcccc}
\hline Location & AIS & HC & OR $(95 \% \mathrm{Cl})$ & $P$ value \\
\hline ICA & $48(52.2)$ & $65(68.4)$ & Reference & \\
MCA & $22(23.9)$ & $8(8.4)$ & $3.72(1.53-9.08)$ & 0.004 \\
A-com & $9(9.8)$ & $7(7.4)$ & $1.74(0.61-5.00)$ & 0.303 \\
P-com & $6(6.5)$ & $9(9.5)$ & $0.90(0.30-2.71)$ & 0.855 \\
VB & $7(7.6)$ & $2(2.1)$ & $4.74(0.94-21.83)$ & 0.059 \\
ACA & $0(0)$ & $4(4.2)$ & - & 0.999
\end{tabular}

Values are number of patients (\%) unless otherwise specified. AIS, acute ischemic stroke; $\mathrm{HC}$, health check-up; $\mathrm{OR}$, odds ratio; $\mathrm{Cl}$, confidence interval; ICA, internal carotid artery; MCA, middle cerebral artery; Acom, anterior communicating artery; P-com, posterior communicating artery; $\mathrm{VB}$, vertebrobasilar artery; $A C A$, anterior cerebral artery.

\section{Independent predictors of UCAs in AIS patients}

Age, female sex, hypertension, diabetes, hyperlipidemia, previous stroke history, smoking, and family history of stroke were analyzed using logistic regression analysis (Table 4). Univariate and multivariate analysis showed that only hypertension $(P=$ 0.028) was correlated with the increased prevalence of UCAs in AIS patients, while other risk factors were not. There was also no difference in the prevalence of UCAs according to ischemic stroke subtype by TOAST classification.

\section{Follow-up data}

The mean follow-up duration was 18.3 months (median 13.0, range 1-48 months) in the AIS group and 17.6 months (median 15.0, range 1-48 months) in the HC group. Eight of the 66 patients $(12 \%)$ in the AIS group were treated by clipping $(n=5)$ or coiling $(n=3)$, and 21 of the $75(28 \%)$ patients in HC group were treated by clipping $(n=12)$ or coiling $(n=9)$. Among the patients 
Table 4. Independent predictors of unruptured cerebral aneurysms in acute ischemic stroke patients

\begin{tabular}{|c|c|c|c|c|}
\hline \multirow{2}{*}{ Predictors } & \multicolumn{2}{|c|}{ Univariate } & \multicolumn{2}{|c|}{ Multivariate } \\
\hline & OR $(95 \% \mathrm{Cl})$ & $P$ value & OR (95\% Cl) & $P$ value \\
\hline Age, per 1-year increase & $1.01(0.99-1.03)$ & 0.142 & $1.01(0.99-1.03)$ & 0.571 \\
\hline Female sex & $1.56(0.97-2.51)$ & 0.067 & $1.82(0.95-3.49)$ & 0.072 \\
\hline Hypertension & $2.09(1.19-3.65)$ & 0.010 & $1.95(1.07-3.53)$ & 0.029 \\
\hline Diabetes & $0.84(0.50-1.44)$ & 0.529 & $0.70(0.40-1.20)$ & 0.189 \\
\hline Hyperlipidemia & $1.48(0.80-2.73)$ & 0.210 & $1.43(0.75-2.71)$ & 0.273 \\
\hline Previous stroke & $1.30(0.73-2.33)$ & 0.372 & $1.15(0.63-2.10)$ & 0.642 \\
\hline Smoking & $0.93(0.57-1.50)$ & 0.749 & $1.46(0.76-2.81)$ & 0.256 \\
\hline Family history of stroke & $0.95(0.42-2.14)$ & 0.908 & $1.11(0.49-2.55)$ & 0.806 \\
\hline TOAST & & 0.235 & & 0.276 \\
\hline Large-artery atherosclerosis & Reference & & Reference & \\
\hline Cardioembolism & $2.12(0.99-4.49)$ & 0.051 & $1.98(0.92-4.22)$ & 0.079 \\
\hline Small-vessel disease & $1.89(0.89-3.99)$ & 0.098 & $2.01(0.97-4.49)$ & 0.060 \\
\hline Other determined etiology & $1.53(0.19-12.73)$ & 0.693 & $1.84(0.21-16.12)$ & 0.581 \\
\hline Undetermined etiology & $2.39(1.12-5.13)$ & 0.024 & $2.31(1.07-4.98)$ & 0.033 \\
\hline
\end{tabular}

who did not undergo treatment, aneurysm rupture occurred in 3 patients in the AIS group and none in the HC group.

\section{Discussion}

The overall prevalence of UCAs in the HC group in the present study was similar to that estimated in a recent systemic review published in 2011, which estimated the overall UCA prevalence to be $3.2 \%$ in a population without comorbidity (mean age, 50 years; $50 \%$ men). ${ }^{9}$ This incidence was notably higher than the 2.3\% reported in a 1998 review. In the 2011 study, the authors suggested that this difference was due to corrections made for age and sex, and to the inclusion of more recent studies with higher quality imaging techniques. ${ }^{9}$ The prevalence reported in previous studies included volunteer participants ${ }^{12,13}$ or patients who underwent brain MRA for various reasons, including diverse neurological conditions. ${ }^{14,17,20,21}$ In contrast, the individuals in the $\mathrm{HC}$ group in our study had no neurologic symptoms or other specific reason for undergoing a brain MRA. Therefore, we used the $\mathrm{HC}$ group as a control for comparison with the AIS group.

We found that the prevalence of UCAs was higher in the AIS group than in the HC group. We hypothesized that this difference was due to the high proportion of risk factors related to cerebral aneurysm in the AIS group. This difference was especially significant in the 7th decade. There is some controversy with respect to the age-related increase in the prevalence of UCAs. Specifically, some studies reported that the prevalence of UCAs steadily increased with age in both genders, ${ }^{7,17}$ whereas other studies found no age-related increase in the prevalence of UCAs. $^{11,20,22}$ Interestingly, we identified an age-related increase in UCA prevalence in the HC group only, which was particularly apparent among females. On the other hand, there was a decreased prevalence of UCAs in the AIS group after peaking in the 7th decade. This discrepancy may be associated with high aneurysmal rupture risk as well as high mortality unrelated to cerebral aneurysms in this age group of ischemic stroke patients. ${ }^{23,24}$ The results of our study also revealed an age-related increase in the size of aneurysms in the AIS group, which was not observed in the HC group. This may be due to the fact that AIS patients have more risk factors associated with growth of the aneurysm.

Besides the higher prevalence and larger aneurysm size observed in the AIS group, some important determinants of eventual cerebral aneurysm rupture were also more common in this group, such as hypertension, smoking, and alcohol consumption. ${ }^{1,25,26}$ Indeed, aneurysmal rupture was more common overall in the AIS group, although long-term follow-up data to confirm this finding were not available. Still, it is unclear whether these findings suggest that ischemic stroke patients have a higher risk of aneurysmal rupture, since the majority of UCAs were small and located in the ICA. ${ }^{3,4,27}$ The reason for the predominant MCA distribution in the AIS group and its clinical significance are both unclear. However, a recent study suggested that the factors affecting the presence of UCAs differ by location. Hokari et al. ${ }^{28}$ suggested that cerebrovascular disease itself as well as hypertension are independent risk factors of MCA aneurysm, and another study demonstrated its association with smoking. ${ }^{29}$ Therefore, we speculate that these risk factors, all of which were more common in the AIS group, could contribute to the high incidence of MCA aneurysms. Some studies found a relationship between cerebral aneurysm and ICA stenosis; ${ }^{30-34}$ however, neither largeartery atherosclerosis nor other subtypes of the TOAST classification were related to the occurrence of UCA in this study. It seems that because stroke is a complex disease influenced by multiple potential etiologies, the TOAST classification does not purely re- 
flect carotid artery stenosis ${ }^{35}$ or the degree of atherosclerosis. Hypertension, which is an important risk factor for cerebral aneurysm, was the only significant factor associated with UCA in the AIS group.

There are several limitations of this study. First, the diagnosis of UCAs was based on 3-dimensional time-of-flight MRA, not conventional angiography, which may have made it more difficult to diagnose small UCAs and to differentiate small aneurysms from junctional dilatations. However, recent studies ${ }^{36-38}$ have described the high diagnostic accuracy of 3-dimensional time-offlight MRA, indicating that it has a high sensitivity of over 95\% as well as a high specificity ranging from 89 to $97 \%$. Furthermore, MRA has been widely used as a validated noninvasive imaging tool without significant radiation hazard. In fact, most of the recent studies conducted on the prevalence of UCAs have used MRA as the evaluation method. Additionally, two independent experienced readers reviewed the MRA images in this study, and a consensus interpretation was made in cases of discrepancy. Second, this was a single hospital-based and ethnically distinct study. Third, we should consider the characteristics of the $\mathrm{HC}$ group that was used as a control group in this study. They differ from the general population in terms of the distributions of age and sex, and they have more health concerns with good control of risk factors. Lastly, this retrospective design did not show the natural history of UCAs in both groups. Thus, a large prospective study is needed to define the natural history of UCAs in ischemic stroke patients compared to the general population.

\section{Conclusions}

In summary, UCAs were more common in AIS patients than in the control group. The mean size of aneurysms in the AIS group was larger than that in the $\mathrm{HC}$ group, and aneurysm size increased significantly with age in the AIS group only. Among the various risk factors evaluated, only hypertension was associated with the increased prevalence of aneurysms in the AIS group. Ultimately, a follow-up study of UCAs in ischemic stroke patients is required to evaluate the precise risk of aneurysmal rupture in order to assist in refining treatment plans.

\section{References}

1. Brisman JL, Song JK, Newell DW. Cerebral aneurysms. N Engl J Med 2006;355:928-939.

2. van Gijn J, Kerr RS, Rinkel GJ. Subarachnoid hemorrhage. Lancet 2007;369:306-318.

3. Wiebers DO, Whisnant JP, Huston J 3rd, Meissner I, Brown RD $J \mathrm{~J}_{1}$ Piepgras DG, et al. Unruptured intracranial aneurysms: nat- ural history, clinical outcome, and risks of surgical and endovascular treatment. Lancet 2003;362:103-110.

4. Morita A, Kirino T, Hashi K, Aoki N, Fukuhara S, Hashimoto N, et al. The natural course of unruptured cerebral aneurysms in a Japanese cohort. N Engl J Med 2012;366:2474-2482.

5. Juvela S, Poussa K, Lehto H, Porras M. Natural history of unruptured intracranial aneurysms: a long-term follow-up study. Stroke 2013;44:2414-2421.

6. Etminan N, Beseoglu K, Barrow DL, Bederson J, Brown RD Jr, Connolly ES Jr, et al. Multidisciplinary consensus on assessment of unruptured intracranial aneurysms: proposal of an international research group. Stroke 2014;45:1523-1530.

7. Rinkel GJ, Djibuti M, Algra $A$, van Gijn J. Prevalence and risk of rupture of intracranial aneurysms: a systematic review. Stroke 1998;29:251-256.

8. Brown RD Jr, Broderick JP. Unruptured intracranial aneurysms: epidemiology, natural history, management options, and familial screening. Lancet Neurol 2014;13:393-404.

9. Vlak MH, Algra A, Brandenburg R, Rinkel GJ. Prevalence of unruptured intracranial aneurysms, with emphasis on sex, age, comorbidity, country, and time period: a systematic review and meta-analysis. Lancet Neurol 2011;10:626-636.

10. Atkinson JL, Sundt TM Jr, Houser OW, Whisnant JP. Angiographic frequency of anterior circulation intracranial aneurysms. J Neurosurg 1989;70:551-555.

11. Vernooij MW, Ikram MA, Tanghe HL, Vincent AJ, Hofman A, Krestin GP, et al. Incidental findings on brain MRI in the general population. N Engl J Med 2007;357:1821-1828.

12. Li MH, Chen SW, Li YD, Chen YC, Cheng YS, Hu DJ, et al. Prevalence of unruptured cerebral aneurysms in Chinese adults aged 35 to 75 years: a cross-sectional study. Ann Intern Med 2013;159:514-521.

13. Muller TB, Sandvei MS, Kvistad KA, Rydland J, Haberg A, Vik A, et al. Unruptured intracranial aneurysms in the Norwegian Nord-Trøndelag Health Study (HUNT): risk of rupture calculated from data in a population-based cohort study. Neurosurgery 2013;73:256-261.

14. Igase K, Matsubara I, Igase M, Miyazaki H, Sadamoto K. Initial experience in evaluating the prevalence of unruptured intracranial aneurysms detected on 3-tesla MRI. Cerebrovasc Dis 2012;33:348-353.

15. Kojima M, Nagasawa S, Lee YE, Takeichi Y, Tsuda E, Mabuchi N. Asymptomatic familial cerebral aneurysms. Neurosurgery 1998:43:776-781.

16. Winn HR, Jane JA Sr, Taylor J, Kaiser D, Britz GW. Prevalence of asymptomatic incidental aneurysms: review of 4568 arteriograms. J Neurosurg 2002;96:43-49.

17. Horikoshi T, Akiyama I, Yamagata Z, Nukui H. Retrospective 
analysis of the prevalence of asymptomatic cerebral aneurysm in 4518 patients undergoing magnetic resonance angiography-when does cerebral aneurysm develop? Neurol Med Chir (Tokyo) 2002;42:105-112.

18. Adams HP, Jr., Bendixen BH, Kappelle U, Biller J, Love BB, Gordon $\mathrm{DL}$, et al. Classification of subtype of acute ischemic stroke. Definitions for use in a multicenter clinical trial. TOAST. Trial of Org 10172 in Acute Stroke Treatment. Stroke 1993;24:35-41.

19. Mizutani T, Miki Y, Kojima H, Suzuki H. Proposed classification of nonatherosclerotic cerebral fusiform and dissecting aneurysms. Neurosurgery 1999;45:253-259; discussion 259-260.

20. Jeon TY, Jeon P, Kim KH. Prevalence of unruptured intracranial aneurysm on MR angiography. Korean J Radiol 2011;12:547553.

21. Park S, Lee DH, Ryu CW, Pyun HW, Choi CG, Kim SJ, et al. Incidental saccular aneurysms on head MR angiography: 5 years' experience at a single large-volume center. J Stroke 2014;16: 189-194.

22. Iwamoto $H$, Kiyohara $Y$, Fujishima M, Kato I, Nakayama $K$, Sueishi $K$, et al. Prevalence of intracranial saccular aneurysms in a Japanese community based on a consecutive autopsy series during a 30-year observation period. The Hisayama study. Stroke 1999;30:1390-1395.

23. Tominari S, Morita A, Ishibashi T, Yamazaki T, Takao H, Murayama $Y$, et al. Prediction model for 3-year rupture risk of unruptured cerebral aneurysms in Japanese patients. Ann Neurol 2015;77:1050-1059.

24. Juvela $S$, Lehto $H$. Risk factors for all-cause death after diagnosis of unruptured intracranial aneurysms. Neurology 2015; 84:456-463.

25. Chalouhi N, Hoh BL, Hasan D. Review of cerebral aneurysm formation, growth, and rupture. Stroke 2013;44:3613-3622.

26. Bacigaluppi S, Piccinelli M, Antiga L, Veneziani A, Passerini T, Rampini $P$, et al. Factors affecting formation and rupture of intracranial saccular aneurysms. Neurosurg Rev 2014;37:1-14.

27. Greving JP, Wermer MJ, Brown RD, Jr., Morita A, Juvela S, Yonekura $M$, et al. Development of the PHASES score for prediction of risk of rupture of intracranial aneurysms: a pooled analysis of six prospective cohort studies. Lancet Neurol 2014;
13:59-66.

28. Hokari M, Isobe M, Imai T, Chiba Y, Iwamoto N, Isu T. The impact of atherosclerotic factors on cerebral aneurysm is location dependent: aneurysms in stroke patients and healthy controls. J Stroke Cerebrovasc Dis 2014;23:2301-2307.

29. Kang HG, Kim BJ, Lee J, Kim MJ, Kang DW, Kim JS, et al. Risk factors associated with the presence of unruptured intracranial aneurysms. Stroke 2015;46:3093-3098.

30. Héman LM, Jongen LM, van der Worp HB, Rinkel GJ, Hendrikse J. Incidental intracranial aneurysms in patients with internal carotid artery stenosis: a CT angiography study and a metaanalysis. Stroke 2009;40:1341-1346.

31. Liang Y, Wang J, Li B. Coexistence of internal carotid artery stenosis with intracranial aneurysm. Int J Stroke 2014;9:306307.

32. Cho YD, Jung KH, Roh JK, Kang HS, Han MH, Lim JW. Characteristics of intracranial aneurysms associated with extracranial carotid artery disease in South Korea. Clin Neurol Neurosurg 2013;115:1677-1681.

33. Chaturvedi S, Sacco RL. How recent data have impacted the treatment of internal carotid artery stenosis. J Am Coll Cardiol 2015;65:1134-1143.

34. Goessens BM, Visseren FL, Kappelle $\sqcup$, Algra A, van der Graaf Y. Asymptomatic carotid artery stenosis and the risk of new vascular events in patients with manifest arterial disease: the SMART study. Stroke 2007;38:1470-1475.

35. Tejada J, Diez-Tejedor $E_{1}$ Hernández-Echebarría L, Balboa 0. Does a relationship exist between carotid stenosis and lacunar infarction? Stroke 2003;34:1404-1409.

36. Li MH, Li YD, Gu BX, Cheng YS, Wang W, Tan HO, et al. Accurate diagnosis of small cerebral aneurysms $\leq 5 \mathrm{~mm}$ in diameter with 3.0-T MR angiography. Radiology 2014;271:553-560.

37. Sailer AM, Wagemans BA, Nelemans PJ, de Graaf R, van Zwam WH. Diagnosing intracranial aneurysms with MR angiography: systematic review and meta-analysis. Stroke 2014;45:119-126.

38. Li MH, Li YD, Tan HQ, Gu BX, Chen YC, Wang W, et al. Contrast-free MRA at 3.0 $\mathrm{T}$ for the detection of intracranial aneurysms. Neurology $2011 ; 77: 667-676$. 
Supplemental Table 1. Characteristics of the patients with unruptured cerebral aneurysm

\begin{tabular}{lccc}
\hline Characteristics & AIS $(n=74)$ & HC $(n=79)$ & $P$ value \\
\hline Age (mean \pm SD), (year) & $67.2 \pm 11.2$ & $57.2 \pm 8.8$ & $<0.001^{*}$ \\
Male & $39(52.7)$ & $35(44.3)$ & 0.30 \\
Hypertension & $57(77)$ & $35(44.3)$ & $<0.001^{*}$ \\
Diabetes & $20(27.0)$ & $14(17.7)$ & 0.166 \\
Hyperlipidemia & $14(18.9)$ & $12(15.2)$ & 0.539 \\
Previous stroke & $16(21.6)$ & $0(0)$ & $<0.001^{*}$ \\
Smokers & $31(41.9)$ & $21(26.6)$ & $0.046^{*}$ \\
Family history of stroke & $13(17.6)$ & $6(7.6)$ & 0.062 \\
\hline
\end{tabular}

Values are number of patients (\%) unless otherwise specified.

AIS, acute ischemic stroke; $\mathrm{HC}$, health check-up.

*Significant value. 
Supplemental Table 2. Size of aneurysm by location

\begin{tabular}{lcccccc}
\hline & ICA & MCA & A-com & P-com & VB & ACA \\
\hline AIS size $(\mathrm{mm})$ & $3.4 \pm 1.7$ & $4.5 \pm 2.9$ & $3.4 \pm 1.3$ & $4.2 \pm 3.4$ & $4.3 \pm 2.5$ & - \\
HC size $(\mathrm{mm})$ & $2.8 \pm 1.0$ & $2.8 \pm 1.8$ & $4.3 \pm 2.4$ & $4.3 \pm 3.0$ & $2.3 \pm 0.4$ & $2.7 \pm 0.9$ \\
Pvalue $^{*}$ & $0.034^{+}$ & 0.139 & 0.328 & 0.955 & 0.316 & - \\
\hline
\end{tabular}

Data are mean \pm SD unless otherwise indicated.

ICA, internal carotid artery; MCA, middle cerebral artery; A-com, anterior communicating artery; P-com, posterior communicating artery; VB, vertebrobasilar artery; $A C A$, anterior cerebral artery; AIS, acute ischemic stroke; $H C$, health check-up.

*For comparison of size of the aneurysms between AIS group and HC group; ${ }^{+}$Significant value. 